\title{
Psychiatric disorders in children with demyelinating diseases of the central nervous system
}

\section{ABSTRACT}

Introduction: The profile of psychiatric disorders associated with multiple sclerosis (MS) may differ in children. We aimed to assess the risk of psychiatric disorders in children with MS and other demyelinating diseases, and vice versa.

Patient and methods: We analysed linked English Hospital Episode Statistics, and mortality data, 1999-2011. Cohorts were constructed of children admitted with MS and other CNS demyelinating diseases. We searched for any subsequent episode of care with psychiatric disorders in these cohorts, and compared to a reference cohort.

Results: Children with CNS demyelinating diseases had an increased rate of psychotic disorders (rate ratio (RR) 5.77 (95\% CI 2.48-11.41)), anxiety, stress-related and somatoform disorders ( $\mathrm{RR}=2.38$ (1.39-3.81)), intellectual disability $(\mathrm{RR}=6.56(3.66-10.84))$, and other behavioural disorders $(\mathrm{RR}=8.99$ (5.13-14.62)). In analysis of the pediatric MS cohort as the exposure, there were elevated rates of psychotic disorders $(R R=10.76(2.93-27.63))$, mood disorders $(R R=2.57$ (1.03-5.31)) and intellectual disability $(\mathrm{RR}=6.08$ (1.25-17.80)). In reverse analyses, there were elevated rates of a recorded hospital episode with CNS demyelinating disease after a previous recorded episode with anxiety, stress-related and somatoform disorders, ADHD, autism, intellectual disability, and other behavioral disorders.

Conclusions: This analysis of a national diagnostic database provides strong evidence for an association between pediatric CNS demyelinating diseases and psychiatric disorders, and highlights a need for early involvement of mental health professionals. 


\section{INTRODUCTION}

Multiple sclerosis (MS) typically onsets in young adulthood (ages 20-40), but can occur at extremes of age. Children with MS (and other demyelinating diseases) are rare, but pediatric-onset MS (prior to age 18) represents 3-5\% of cases. ${ }^{1}$ There is an increased risk of cognitive impairment and psychiatric disorders (primarily mood and anxiety disorders) in adults with MS, and their presence has been associated with a worse quality of life and medication adherence. ${ }^{2-3}$ The profile of psychiatric diseases may differ in children where the pathophysiology occurs during a key period of central nervous system (CNS) development, and their study may be important in understanding the impact of MS more broadly. The establishment of multi-institutional collaborative initiatives has recently facilitated the study of pediatric MS cohorts, but studies of psychiatric disorders have often been limited by small sample sizes, a lack of control cohorts, selection bias in recruitment, or limited follow-up (and consequent cross-sectional study design and poor characterization of a temporal relationship).${ }^{4-9}$ Characterizing the risk of psychiatric outcomes in these children is necessary to inform clinicians and parents, and to highlight a potential need for increased psychiatric awareness/monitoring/support in this vulnerable group. We aimed to assess the risk of psychiatric disorders in children with MS and other demyelinating diseases, and vice versa, using linked English national Hospital Episode Statistics.

\section{METHODS}

\section{Population and data}

A retrospective cohort study was conducted using linked English National Hospital Episode Statistics (HES) and mortality, 1999-2011. HES are recorded abstracts of each episode of day-case or inpatient care in all English National Health Service hospitals. Cohorts were constructed of children with MS and other demyelinating diseases of the CNS by identifying the first recorded episode of day-case or inpatient care prior to age 18 for any of the included conditions, in any diagnostic position, whether as the principal diagnosis or otherwise (the "exposure" cohorts, ICD10 codes multiple sclerosis G35; other acute disseminated demyelination G36; other demyelinating diseases of the central nervous system G37; and acute disseminated encephalitis G04.0). A constructed reference cohort incorporated individuals with an initial admission for a range of various other minor medical and surgical conditions (Table 1 
footnotes). Any individual with a co-occurring or prior admission with any of the psychiatric outcomes at the first time of care for the demyelinating disease or reference condition was excluded from the analysis. We searched for any subsequent episode of NHS care, or death from, psychiatric disorders in these cohorts (the "outcome" cohorts ICD10 codes suicide attempt X60-X84, Y10-Y34; psychotic disorders F20-F29 (schizophrenia, schizotypal disorder, persistent delusional disorders, acute and transient psychotic disorders, and schizoaffective disorders, other nonorganic psychotic disorders, and unspecified nonorganic psychosis); mood disorders F30-F39; anxiety, dissociative, stress-related and somatoform disorders F40-F45, F48; eating disorders F50; psychoactive substance misuse F10-F19; attention-deficit hyperactivity disorder (ADHD) F90; autism spectrum disorder F84; intellectual disability F70-F79; other behavioral disorders F91-F98; or any of the above). The rates of psychiatric disorders in the reference cohort are considered to represent that of the general population (allowing for migration in and out of it, as data on migration is not included in these datasets). Using this methodology we also conducted reverse analysis of the risk of demyelinating diseases in children with prior psychiatric disorders.

\section{Statistical methods}

The date of entry into each cohort was counted as the date of first admission for any exposure, or reference, condition. The first recorded episode of a psychiatric disorder, death, or end of data collection in 2011 (whichever came first), was considered as the date of exit. The rates of psychiatric disorders were calculated, stratified, and standardized by age, sex, calendar year of first recorded admission of the exposure condition, region of residence, and quintile of patients' Index of Deprivation score (a standard English measure of socio-economic status). The indirect method of standardization was used, with the combined exposure and reference cohort as the standard population. Stratum-specific rates in the standard population were applied to the number of person-days in each stratum in each exposure cohort and then, separately, to those in the same stratum in the reference cohort, to obtain the expected number of people with each psychiatric disorder in each stratum of the exposure and reference cohorts. Observed and expected numbers were then summed across all strata to give totals for all strata combined. Rate ratios (RR) were calculated using the formula $\left(\mathrm{O}^{\mathrm{DEM}} / \mathrm{E}^{\mathrm{DEM}}\right) /\left(\mathrm{O}^{\mathrm{REF}} / \mathrm{E}^{\mathrm{REF}}\right)$, where $\mathrm{O}$ and $\mathrm{E}$ are the observed and expected numbers of cases of the psychiatric disorders in the demyelinating disease and reference cohorts, respectively. The confidence intervals for the RR of the psychiatric disorders and $\chi^{2}$ statistics for their significance were calculated, for which the more detailed methods of analysis have been described elsewhere. ${ }^{10-11}$

\section{RESULTS}


Pediatric CNS demyelinating diseases before psychiatric disorders

201 children aged 0-17 entered the MS cohort, 1097 children entered the demyelinating diseases cohort (including MS), and over 1.1 million children entered the reference cohort. Among the children in the MS cohort, $62.4 \%$ had at least two hospital episodes with MS coded, 51.3\% had at least three hospital episodes with MS coded, and $16.9 \%$ had 10 or more MS episodes coded. Children with a demyelinating disease had a significantly increased rate of psychotic disorders (standardized RR 5.77 (2.48-11.41), p<0.001), anxiety, stress-related and somatoform disorders (RR 2.38 (1.39-3.81), $\mathrm{p}<0.001$ ), intellectual disability (RR 6.56 (3.66-10.84), $\mathrm{p}<0.001)$, other behavioral disorders (RR 8.99 (5.13-14.62), $\mathrm{p}<0.001$ ), and an elevated rate of any of the included psychiatric disorders (RR 1.56 (1.24-1.94), $\mathrm{p}=0.0001)$, Table 1a. Analysis including episodes of care for psychiatric disorders which occurred with a first known record at least one year, and at least five years, from the first recorded episode of demyelinating disease, demonstrated that the association remained significant for all aforementioned outcomes with a 1 year minimum interval (Table $1 \mathrm{~b}$ ), and remained significant for psychotic disorders, intellectual disability and other behavioral disorders with a minimum 5 year interval (Table 1c). In analyses including only the pediatric MS cohort as the exposure (smaller sample size), there were significantly elevated rates of psychotic disorders (RR 10.76 (2.93-27.63), p<0.001), mood disorders (RR 2.57 (1.035.31), $\mathrm{p}=0.022)$ and intellectual disability (RR 6.08 (1.25-17.80), $\mathrm{p}=0.004)$, Table 2). In analysis limited to psychiatric disorders first recorded at least one year following the first MS episode, despite smaller numbers, associations remained significant for MS and psychotic disorders (RR 8.95 (1.84-26.24), p<0.001), and MS and intellectual disability (RR $6.68(1.38-19.56), \mathrm{p}=0.002)$.

Pediatric psychiatric disorders before CNS demyelinating diseases

Further, in reverse analysis of the risk of demyelinating diseases in children with psychiatric disorders (Table 3), there were significant associations for subsequent demyelinating disease in children with anxiety, stress-related and somatoform disorders (RR 3.15 (1.70-5.39), p<0.001), ADHD (RR 3.88 (1.75-7.48), p<0.001), autism (RR 3.80 (2.056.50), $\mathrm{p}<0.001$ ), intellectual disability (RR 6.33 (2.86-12.21), $\mathrm{p}<0.001$ ), other behavioral disorder (RR 8.30 (5.1712.75), $\mathrm{p}<0.001$ ), and for any of the included psychiatric disorders (RR $2.15(1.70-2.72), \mathrm{p}<0.001)$. These associations remained significant when only including cases of demyelinating disease for which the first hospital episode occurred at least 1 year after the first episode for the psychiatric disorder; anxiety, stress-related and somatoform disorders (RR 2.60 
(1.17-5.04), p=0.08), ADHD (RR 3.33 (1.21-7.38), p=0.007), autism (RR 2.90 (1.24-5.82), $\mathrm{p}=0.005)$, intellectual disability (RR 3.98 (1.28-9.44), p<0.001), other behavioral disorder (RR 7.19 (4.11-11.79), p<0.001), and for any of the included psychiatric disorders (RR 1.92 (1.47-2.50), $\mathrm{p}<0.001)$. For the reverse analysis, numbers were too small for meaningful data of MS only, for data on a 5-year interval between exposure and outcome.

\section{TABLE 1}

TABLE 2

TABLE 3

\section{DISCUSSION}

This large population-based study presents strong evidence of a positive association between childhood CNS demyelinating diseases and psychiatric disorders, including between demyelinating diseases and psychotic disorders, anxiety, stress-related and somatoform disorders, intellectual disability, and other behavioral disorders. In the study of only MS, an additional significant association was observed between MS and mood disorders. Reverse analysis, to consider the possibility of reverse causality, demonstrates that the observed associations are found in either direction, with a few exceptions; psychotic disorders were not significant preceding demyelinating disease (but this finding may reflect the smaller number of cases). Further, ADHD and autism were found to be associated with subsequent demyelinating disease (including when only incorporating cases of demyelinating diseases identified at least one year after the earliest known ADHD or autism hospital episode). This may in part reflect that ADHD and autism tend to be diagnosed relatively early in life, whereas psychotic disorders tend to be diagnosed in adolescence or young adulthood; alternatively, these associations may reflect the presence of underlying inherited or metabolic disorders.

Our findings support previous work suggesting an increased risk of psychiatric disorders in individuals with adult and pediatric MS. Psychiatric examination of 45 pediatric MS patients identified 25 with a psychiatric disorder, of which two thirds had more than one diagnosis, most commonly anxiety $(\mathrm{n}=15)$, ADHD $(\mathrm{n}=12)$ and mood disorders $(\mathrm{n}=11) .{ }^{4} \mathrm{~A}$ study of 23 children with demyelinating diseases found a similar $48 \%$ prevalence of psychiatric disorders. ${ }^{5}$ Behavioral disturbances in these children have not been well characterized but many potential contributing factors exist, such as disease-associated poor school attendance, low self-esteem or uncertainty about the nature of the disease. ${ }^{6}$ Cognitive impairment has been widely reported in MS, but fewer studies exist incorporating children, a possibly particularly susceptible group undergoing a demyelinating process concomitantly with CNS development. ${ }^{6,12}$ A cohort of 
childhood MS cases in Italy compared cognitive and psychosocial functioning at a baseline evaluation and at two years subsequently. They classified $75 \%$ of included cases as having deteriorating cognitive performance over time, highlighting a need for early identification and treatment to address progression of psychiatric symptoms. ${ }^{7-8}$ Measurement of cognitive functioning in 231 children with MS and clinically isolated syndrome (CIS) from US centers reported cognitive impairment of $35 \%$ of children with MS and $18 \%$ of those with CIS. ${ }^{13}$

The increased rate of psychotic disorders following demyelinating disease (and MS specifically), and the increased rate of demyelinating disease following autism, observed in this study, are particularly intriguing as, to our knowledge, these have not before been reported in a pediatric population. A postulated increased rate of bipolar disorder has previously been reported among adults with MS, and a systematic review considered the study of psychotic disorders in MS to have been inconsistent and in need of further work. ${ }^{14-15}$ It raises intriguing questions about the underlying pathophysiology of psychotic disorders, and whether this association represents shared risk factors (environmental or genetic predisposition), a maladaptive response to high pressures of dealing with chronic disease in childhood, or shared causal mechanistic pathways. It is important to consider the possibility of organic causes of psychosis in patients with MS, in addition to potential treatment-induced psychosis. Psychosis in patients with MS may be putatively attributed to temporal lobe brain lesions, ${ }^{16}$ corticosteroid induced psychosis, ${ }^{17}$ or the proposition that psychotic disorders and MS may share an autoimmune pathophysiology. The latter is supported by analysis of Danish National Registers which have reported a $45 \%$ increased risk of schizophrenia in individuals with a personal history of any autoimmune disease, as well as an increased risk of schizophrenia in individuals with a first-degree relative with onset of an autoimmune disease before the case of schizophrenia. ${ }^{18-19}$

It is possible, both in the case of autism followed by demyelinating disease, and more broadly, that the diagnoses do not represent separate disease entities. Rather, some cognitive and behavioral features which form part of a diagnosis of autism may represent the symptomatic profile of the early stages of CNS demyelinating disease such as inherited white matter diseases. Our ability to further evaluate this potential association is limited not only by the inability to review the medical records in detail, but also by the lack of capacity to formally review the MRI studies. Further, we also note that cognitive impairment, depression, and other psychiatric symptoms may be prodromal early manifestations of MS. We hope our findings may prompt clinicians with young patients with psychiatric presentations to consider the possibility of an underlying demyelinating disease process, and to carefully monitor patients with CNS demyelinating diseases for psychiatric presentations as part of a holistic care approach for such patients.

Strengths of this study include the use of a large dataset and a person-based cohort design to undertake an otherwise 
logistically difficult investigation. Limitations include the possibility of surveillance bias, potentially as a consequence of patients with greater contact with healthcare services. We tried to minimize the possibility of surveillance bias by doing analyses only including individuals with a first record of the outcome at least 1 year, and 5 years, after first record of the exposure (and, of note, our findings remain strong). We consider that where numbers of cases in this study were small, these findings may be considered as "the best possible" utilizing national routine data from a population of more than 50 million. Therefore, it is likely that we detected significant results for the strongest associations, but that further associations may exist, as is suggested by the data presented. This may also be a reflection of the fact that only day-case and inpatient hospital data were used (some disorders may never be coded outside primary care), and individuals with the studied psychiatric outcomes (in both the exposure and reference cohort) may over-represent those at the more severe end of disease spectrum. It follows that, though rate ratios for the observed associations are high, the true prevalence of psychiatric disorders among individuals with demyelinating diseases is almost certainly higher than is suggested by our data. Further, we note that where the "expected" number of cases is very low, a marked increase in rate ratio may be observed from the presence of only a few patients above the "expected" value.

It is also worth considering that the true number of pediatric MS cases is likely higher than the 201 identified in the MS cohort, as MS in children is a difficult diagnosis to make, and some of these children will be coded with other CNS demyelinating diagnoses (and included in the demyelinating diseases cohort) with a diagnosis of MS made at an older age. An important limitation is that unfortunately diagnostic detail, and detailed data on clinical characteristics was not available. We are therefore unable to confirm diagnoses, which would necessitate review of the patient records. Information on other potential shared risk factors (beyond for example socioeconomic status which could be obtained through routinely collected data), was also not available. In light of this, as this study draws upon routinely collected national data based on disease codes by clinicians, we are unable to verify the diagnoses and diagnostic inaccuracy remains possible.

Different diagnostic criteria may have been used through the time period covered by HES, and no clinical or imaging review is possible to ensure accuracy of diagnostic coding. This may lead to an underestimated rate of demyelinating or psychiatric diseases, as well as inclusion of some false positive subjects. Nonetheless, we consider it likely that coding a diagnosis of MS or a psychiatric disorder on the medical record of a child would not be done by a clinician if not in accordance with the appropriate diagnostic criteria in practice in the UK. We have also presented our result within the categories of both 'MS' and 'CNS demyelinating diseases' more broadly due to consideration of the fact that MS is a 
very difficult clinical diagnosis in children, where the presentation is often more atypical. As an indicator of likely MS, as stated in Results, we found that out of the $201 \mathrm{MS}$ children, $64.2 \%$ had at least two recorded hospital episodes with MS, 51.3\% had at least 3 hospital episodes with MS, and $16.9 \%$ had 10 or more hospital episodes with MS. This data should be considered in the context of the specific difficulty of diagnosing MS in children; but it makes it likely that the majority of children probably did have MS.

Another limitation of the study lies in the unavailability of data on investigations of differential diagnosis, likely in part due to a lack of testing for some conditions earlier in the study period. For example, disease associated with aquaporin-4 antibodies have been associated with psychiatric comorbidity and may be included in patients with MS or other CNS demyelinating diseases. ${ }^{20}$ Similarly, presence of NMDA-receptor antibodies has been associated with psychiatric disease, has been described as a clinical phenotype suggestive of CNS demyelinating disease in children, and may in the past have been coded as such. ${ }^{21}$ Limited by end of data collection in December 2011, our data are also unable to reflect what the risks may be in a study of longer duration of follow-up, where for example some psychiatric disorders may become more prominent.

Moreover, the study utilizes prevalent cases based on first recorded hospital episode, and is not a follow-up from first point of diagnosis. It is possible that some cases with MS or other CNS demyelinating disorders are not included due to not having encountered day-case or inpatient hospital care within the study period.

In conclusion, this study reports a significantly increased rate of psychiatric disorders in children with demyelinating diseases, and to our knowledge provides the first reported association between childhood demyelinating diseases and psychotic disorders. We support current clinical practice aiming to provide a holistic management approach, and encourage specific measures aiming to identify a variety of psychiatric disorders and involvement of mental health professionals, as part of multi-disciplinary routine care. Early intervention and establishment of coping strategies for the child and parents may be critical in limiting the impact on academic performance and motivation. Further work should be done to determine whether these associations are all specific to childhood demyelinating disorders, or whether some are also found in childhood in other chronic disabling diseases. Prospective longitudinal studies are needed to characterise more carefully the temporal relationship between psychiatric disorders and demyelinating diseases, the progression of psychiatric disorders in these children, and how these correlate with clinical disease activity. 


\section{ACKNOWLEDGEMENTS AND FUNDING}

The Health and Social Care Information Centre provided data on HES, and the Office for National Statistics provided data on death registrations. The Oxford record-linkage group undertook linkage of the records in constructing a timesequenced record of successive care episode (or death, if applicable) for each person. The building of the linked datasets, and the development of the analytical software used to study disease associations, was funded by the English National Institute for Health Research (ref RNC/035/002). The build and use of the datasets received ethical approval from the Central and South Bristol research Ethics Committee (ref 04/Q2006/176). RG's salary is part-funded by Public Health England. This study had no specific funding. The funders had no role in study design, data collection, data analysis, data interpretation, writing of the report or for the decision to submit for publication. The views expressed in the paper do not necessarily reflect those of the funding bodies.

\section{COMPETING INTERESTS}

All authors declare no conflicts of interest. All authors report no relevant disclosures.

\section{AUTHOR CONTRIBUTIONS}

JP proposed the study and wrote the first draft of the manuscript. RG and MJG designed the analysis, which was conducted by RG. MJG is the guarantor of the study. All authors contributed to the interpretation of the data. All authors edited the manuscript for important intellectual content.

\section{REFERENCES}

1. Chitnis T, Glanz B, Jaffin S, Healy B. Demographics of pediatric-onset multiple sclerosis in an MS center population from the Northeastern United States. Mult Scler 2009;15:627-631.

2. Mohr DC, Goodkin DE, Likosky W, et al. Treatment of depression improves adherence to interferon beta-1b therapy for multiple sclerosis. Arch Neurol 1997;54:531-533.

3. Jønsson A, Dock J, Ravnborg MH. Quality of life as a measure of rehabilitation outcome in patients with 
multiple sclerosis. Acta Neurol Scand 1996;93(4):229-35.

4. Weisbrot D, Charvet L, Serafin D, et al. Psychiatric diagnoses and cognitive impairment in pediatric multiple sclerosis. Mult Scler 2014;20(5):588-93.

5. Weisbrot DM, Ettinger AB, Gadow KD, et al. Psychiatric comorbidity in pediatric patients with demyelinating disorders. J Child Neurol 2010;25:192-202.

6. MacAllister WS, Boyd JR, Holland NJ, et al. The psychosocial consequences of pediatric multiple sclerosis. Neurology 2007;68:S66-69.

7. Amato MP, Goretti B, Ghezzi A, et al. Cognitive and psychosocial features of childhood and juvenile MS. Neurology 2008;70:1891-1897.

8. Amato MP, Goretti B, Ghezzi A, et al. Cognitive and psychosocial features in childhood and juvenile MS: two-year follow-up. Neurology 2010;75:1134-1140.

9. Goretti B, Ghezzi A, Portaccio E, Amato MP. Psychosocial issue in children and adolescents with multiple sclerosis. Neurol Sci 2010;31:467-470.

10. Breslow NE, Day NE. Statistical methods in cancer research. Volume II - The design and analysis of cohort studies. IARC Sci Publ 1987;82:1-406.

11. Gold J, Goldacre R, Maruszak H, et al. HIV and lower risk of multiple sclerosis: Beginning to unravel a mystery using a record-linked database study. J Neurol Neurosurg Psychiatry 2014;86(1):9-12.

12. MacAllister WS, Belman AL, Milazzo M, et al. Cognitive functioning in children and adolescents with multiple sclerosis. Neurology 2005;64(8):1422-5.

13. Julian L, Serafin D, Charvet L, et al. Cognitive impairment occurs in children and adolescents with multiple sclerosis: results from a United States network. J Child Neurol 2013;28(1):102-7.

14. Marrie RA, Reingold S, Cohen J, et al. The incidence and prevalence of psychiatric disorders in multiple sclerosis: A systematic review. Mult Scler 2015;21(3):305-317.

15. Johansson V, Lundholm C, Hillert J, et al. Multiple sclerosis and psychiatric disorders: comorbidity and sibling risk in a nationwide Swedish cohort. Mult Scler 2015;20(14):1881-91.

16. Yadav R, Zigmond AS. Temporal lobe lesions and psychosis in multiple sclerosis. BMJ Case Rep 2010;2010.

17. The Boston Collaborative Drug Surveillance Program. Acute adverse reactions to prednisone in relation to dosage. Clin Pharmacol Ther 1972;13:694-8.

18. Eaton WW, Byme M, Ewald H, Mors O, Chen CY, Agerbo E, Mortensen PB. Association of schizophrenia and autoimmune diseases: linkage of Danish national registers. Am J Psychiatry 2006;163(3):521-8.

19. Eaton WW, Pedersen MG, Nielsen PR, Mortensen PB. Autoimmune diseases, bipolar disorder, and non- 
affective psychosis. 2010;12(6):638-46.

20. Popescu BF, Guo Y, Jentoft ME, et al. Diagnostic utility of aquaporin-4 in the analysis of active demyelinating lesions. Neurology 2015;84(2):148-58.

21. Titulaer MJ, Höftberger R, Iizuka T, et al. Overlapping demyelinating syndromes and anti-N-methyl-Daspartate receptor encephalitis. Ann Neurol 2014;75(3):411-28. 
Table 1. Rate ratios and $95 \%$ confidence intervals (CIs) for psychiatric disorders in children with CNS demyelinating diseases

\begin{tabular}{|c|c|c|c|c|}
\hline Outcome & Observed & Expected & Rate Ratio (95\% CI) & P-value \\
\hline Self-harm & 21 & 22.3 & $0.94(0.58-1.44)$ & 0.866 \\
\hline Psychotic disorders & 8 & 1.4 & $5.77(2.48-11.41)$ & $<0.001$ \\
\hline Mood disorders & 17 & 11.1 & $1.54(0.9-2.47)$ & 0.101 \\
\hline Anxiety, stress-related and somatoform disorders & 17 & 7.2 & $2.38(1.39-3.81)$ & $<0.001$ \\
\hline Eating disorders & 2 & 1.2 & $1.65(0.2-5.98)$ & 0.793 \\
\hline Psychoactive substance misuse & 22 & 22.3 & $0.99(0.62-1.5)$ & 0.961 \\
\hline ADHD & 4 & 2.3 & $1.74(0.48-4.47)$ & 0.426 \\
\hline Autism & 4 & 3.7 & $1.09(0.3-2.78)$ & 0.923 \\
\hline Intellectual disability & 15 & 2.3 & $6.56(3.66-10.84)$ & $<0.001$ \\
\hline Other behavioural disorder & 16 & 1.8 & $8.99(5.13-14.62)$ & $<0.001$ \\
\hline Any of the above & 80 & 51.2 & $1.56(1.24-1.94)$ & 0.0001 \\
\hline \multicolumn{5}{|c|}{ Outcome at least 1 year after first exposure admission } \\
\hline Self-harm & 17 & 18.6 & $0.91(0.53-1.46)$ & 0.791 \\
\hline Psychotic disorders & 7 & 1.3 & $5.37(2.16-11.11)$ & $<0.001$ \\
\hline Mood disorders & 14 & 10 & $1.4(0.77-2.36)$ & 0.265 \\
\hline Anxiety, stress-related and somatoform disorders & 14 & 6.1 & $2.31(1.26-3.87)$ & 0.003 \\
\hline Eating disorders & 1 & 1.1 & $0.94(0.02-5.26)$ & 0.67 \\
\hline Psychoactive substance misuse & 20 & 20.4 & $0.98(0.6-1.52)$ & 0.975 \\
\hline ADHD & 3 & 2 & $1.51(0.31-4.42)$ & 0.716 \\
\hline Autism & 4 & 3.2 & $1.26(0.34-3.22)$ & 0.859 \\
\hline Intellectual disability & 14 & 2 & $6.9(3.77-11.6)$ & $<0.001$ \\
\hline Other behavioural disorder & 10 & 1.4 & $7.19(3.44-13.25)$ & $<0.001$ \\
\hline Any of the above & 63 & 44 & $1.43(1.1-1.83)$ & 0.005 \\
\hline \multicolumn{5}{|c|}{ Outcome at least 5 years after first exposure admission } \\
\hline Self-harm & 4 & 7.7 & $0.52(0.14-1.32)$ & 0.243 \\
\hline Psychotic disorders & 4 & 0.7 & $5.39(1.47-13.88)$ & 0.001 \\
\hline Mood disorders & 4 & 5.4 & $0.74(0.2-1.91)$ & 0.705 \\
\hline Anxiety, stress-related and somatoform disorders & 6 & 3.2 & $1.87(0.69-4.08)$ & 0.201 \\
\hline Eating disorders & 0 & 0.5 & $0(0-7.72)$ & 0.976 \\
\hline Psychoactive substance misuse & 8 & 11.9 & $0.67(0.29-1.32)$ & 0.321 \\
\hline ADHD & 1 & 0.9 & $1.09(0.03-6.07)$ & 0.662 \\
\hline Autism & 2 & 1.5 & $1.31(0.16-4.72)$ & 0.979 \\
\hline Intellectual disability & 4 & 1 & $4.04(1.1-10.36)$ & 0.012 \\
\hline Other behavioural disorder & 3 & 0.5 & $6.1(1.26-17.88)$ & 0.004 \\
\hline Any of the above & 21 & 21.4 & $0.98(0.61-1.5)$ & 0.989 \\
\hline
\end{tabular}


Conditions used in the reference cohort: squint, otitis externa/media, varicose veins, haemorrhoids, upper respiratory tract infections, deflected nasal septum, nasal polyp, teeth disorders, inguinal hernia, in-growing toenail and other diseases of nail, sebaceous cyst, internal derangement of knee, bunion, selected limb fractures, dislocations, sprains and strains, superficial injury and contusion, tonsillectomy, adenoidectomy, appendicectomy, hip replacement, knee replacement. 
Table 2. Rate ratios and $95 \%$ confidence intervals (CIs) for psychiatric disorders in children with multiple sclerosis

\begin{tabular}{|lcccc|}
\hline Outcome & Observed & Expected & $\begin{array}{c}\text { Rate Ratio (95\% } \\
\text { CI) }\end{array}$ & $\begin{array}{c}\text { P- } \\
\text { value }\end{array}$ \\
\hline Self-harm & 2 & 5.3 & $0.38(0.05-1.36)$ & 0.221 \\
Psychotic disorders & 4 & 0.4 & $10.76(2.93-27.63)$ & $<\mathbf{0 . 0 0 1}$ \\
Mood disorders & 7 & 2.7 & $2.57(1.03-5.31)$ & $\mathbf{0 . 0 2 2}$ \\
Anxiety, stress-related and somatoform disorders & 3 & 1.7 & $1.73(0.36-5.05)$ & 0.563 \\
Eating disorders & 1 & 0.3 & $3.6(0.09-20.09)$ & 0.674 \\
Psychoactive substance misuse & 7 & 5.3 & $1.32(0.53-2.73)$ & 0.597 \\
ADHD & 0 & 0.3 & $0(0-12.49)$ & 0.707 \\
Autism & 2 & 0.5 & $4.31(0.52-15.59)$ & 0.128 \\
Intellectual disability & 3 & 0.5 & $6.08(1.25-17.8)$ & $\mathbf{0 . 0 0 4}$ \\
Other behavioural disorder & 1 & 0.2 & $5.19(0.13-28.96)$ & 0.484 \\
Any of the above & 18 & 11.7 & $1.54(0.91-2.44)$ & 0.089 \\
\hline
\end{tabular}


Table 3. Rate ratios and $95 \%$ confidence intervals (CIs) for CNS demyelinating diseases in children with psychiatric disorders

\begin{tabular}{|lcccc|}
\hline Exposure & Observed & 'Expected & $\begin{array}{c}\text { Rate Ratio (95\% } \\
\text { CI) }\end{array}$ & $\begin{array}{c}\text { P- } \\
\text { value }\end{array}$ \\
\hline Self-harm & 35 & 29 & $1.24(0.84-1.76)$ & 0.281 \\
Psychotic disorders & 2 & 1.6 & $1.22(0.15-4.45)$ & 0.912 \\
Mood disorders & 10 & 5.8 & $1.74(0.82-3.25)$ & 0.127 \\
Anxiety, stress-related and somatoform disorders & 14 & 4.6 & $3.15(1.70-5.39)$ & $<\mathbf{0 . 0 0 1}$ \\
Eating disorders & 4 & 2.3 & $1.78(0.48-4.61)$ & 0.41 \\
Psychoactive substance misuse & 11 & 15.5 & $0.70(0.34-1.27)$ & 0.292 \\
ADHD & 9 & 2.4 & $3.88(1.75-7.48)$ & $<\mathbf{0 . 0 0 1}$ \\
Autism & 14 & 3.8 & $3.80(2.05-6.50)$ & $<\mathbf{0 . 0 0 1}$ \\
Intellectual disability & 9 & 1.5 & $6.33(2.86-12.21)$ & $<\mathbf{0 . 0 0 1}$ \\
Other behavioural disorder & 23 & 3 & $8.30(5.17-12.75)$ & $<\mathbf{0 . 0 0 1}$ \\
Any of the above & 113 & 64.3 & $2.15(1.70-2.72)$ & $<\mathbf{0 . 0 0 1}$ \\
\hline
\end{tabular}

